\title{
Nanoparticle Polydispersity Can Strongly Affect In Vitro Dose
}

\author{
Laura Rodriguez-Lorenzo, Barbara Rothen-Rutishauser, Alke Petri-Fink, \\ and Sandor Balog*
}

\begin{abstract}
When nanomaterials meet the biological world, the cellular interaction of nanoparticles is routinely assessed in in vitro systems. Establishing doseresponse relationships requires that the dose of nanoparticles delivered to the cell is accurate and precise. Nanoparticles as such or coated with high molecular-weight compounds are rarely uniform and the influence of heterogeneity, including polydispersity both in size and mass density, on the delivered dose is never studied before. Furthermore, a probabilistic term describing particle adherence to cells is introduced and the importance is discussed. By tracing the movement of discrete particles via modeling, it is found that the influence of heterogeneity cannot be neglected when the average particle size promotes settling over diffusion. However, the influence of polydispersity on the delivered cellular dose is less critical for particulate systems whose mean size promotes diffusion. The influence of a non-instantaneous particle association to the cell is negligible for particles whose motion is dominated by settling, but it is relevant for small particles whose motion is governed by diffusion.
\end{abstract}

at least three elementary processes that are relevant for in vitro dose: (i) delivery of NPs to the cell, (ii) adherence to the cell membrane, and (iii) internalization. Any of these processes can be a rate-limiting factor. Awareness concerning the peculiarities of NP transport in fluids and the relevance to liquid-based in vitro assays is on the rise, as shown by the steadily increasing number of research papers adopting the concept of particokinetics proposed by Teeguarden et al. ${ }^{[20,25]}$ for interpreting dose-response curves. The concept of particokinetics, describing uniform particles, ${ }^{[20,25]}$ challenges the still-reigning paradigm that the dose to which adherent cells are exposed to at the bottom of the cell-culture dish is proportional to the concentration of NPs in the suspension. However, the concentration of NPs in suspension defines only the

\section{Introduction}

Nanomaterials science has emerged as a field that impacts virtually all sectors of human life: electronics and IT applications, manufacturing and materials, and healthcare technologies. The growing interest in nanotechnologies also resulted in the development of new scientific disciplines such as "nanomedicine," $[1-4]$ as well as raised concerns about the health risks of manufactured nanomaterials and their environmental impact. $^{[5-10]}$ Significant research efforts in the field of nanomaterials have resulted in nanoparticles (NPs) designed in nearly all sizes and shapes. ${ }^{[11-15]}$

In the last decade, much work has focused on elucidating the impact of physicochemical properties of NPs (e.g., size, surface charge, hydrophobicity, or shape) on their subsequent cellular interaction. In vitro systems are routinely used to assess the cellular interaction of NPs on a single-cell level to reveal mechanistic insights. ${ }^{[16-21]}$ It is widely accepted that there are

Dr. L. Rodriguez-Lorenzo, Prof. B. Rothen-Rutishauser,

Prof. A. Petri-Fink, Dr. S. Balog

Adolphe Merkle Institute

University of Fribourg

1700 Fribourg, Switzerland

E-mail: sandor.balog@unifr.ch administered dose, and the above paradigm is strictly valid only when all NPs in the suspension have completely settled, in which case the delivered dose is equal to the administered dose. The ultimate fate of NPs in a fluid is eventually dictated by its mass density, i.e., NPs will settle if their mass density is greater than that of the fluid. The impact of NP size and mass density on transport has been known for a long time ${ }^{[26-29]}$ : in general, sedimentation is dominant for large NPs of "higher" mass density, while diffusion dominates the transport for small NPs of "lower" mass density. The kinetics of a particle is quantified by the diffusion coefficient (the Stokes-Einstein equation and the settling velocity, Stokes' law). ${ }^{[26]}$ Depending on (i) the hydrodynamic radius of the NPs, (ii) the mass density difference between the particle and the fluid, (iii) the concentration of NPs in the colloidal suspension, (iv) the total volume of the colloidal suspension administrated to the cell culture, and (v) the cross-sectional area of the cell-culture well, temperature and dynamic viscosity of the suspending fluid, the delivery time may be as short as minutes and as long as days in certain cases. The model for the transport and dosimetry of NPs developed by Hindertliter et al. ${ }^{[20]}$ has shown that already uniform NPs present examples of nontrivial dose-time courses. In general, uniform or monodisperse NPs are defined as NPs that present no more than $10 \%$ dispersion in their size distribution. ${ }^{[30]}$ Dynamic changes in the dispersion state of NPs have been shown to significantly complicate theoretical models, which aim to predict dosimetry. ${ }^{[17,31-36]}$ 
Models employ idealized NPs to predict dosimetry, however in vitro, many studies involve heterogeneous suspensions of NPs both in size and in shape. ${ }^{[36-40]}$ The term "monodispersity" is therefore used rather generously, in particular when referring to commercially available nanopowders, particles synthesized on an ultra-large scale, and anthropogenic (incidental) as well as naturally occurring particulate nanomaterials. ${ }^{[41-43]}$ These examples of NPs may be highly heterogeneous both in size and morphology when compared with the engineered NPs found in the biological and/or medical field. In addition, NPs are frequently coated by, e.g., synthetic polymers to prevent aggregation and to allow subsequent surface derivatization, ${ }^{[15,44]}$ or interact strongly with natural biopolymers. ${ }^{[4-48]}$ Yet, the size and influence of surface-bound molecules on NPs is often underestimated. In fluid, the polymer covering a NP forms a shell and defines the hydrodynamic radius, and modifies the effective mass density relevant to settling. ${ }^{[49]}$ Accordingly, a nonuniform or highly polydisperse coating is expected to result in an overall heterogeneity, whereby both size and effective mass density are polydipserse-even if the core NP is per se monodisperse. ${ }^{[49]}$ A recent review by Pelaz et al. ${ }^{[50]}$ has addressed the monodispersity paradigm. The authors convincingly show how uniformity might be required for some applications (e.g., lightemitting quantum-dot structures, where the emission wavelength strongly depends on the size of the quantum dot), while other applications are rather indifferent to nonuniformity. However, when estimating the distributions of diffusion coefficient and settling velocity, respectively, polydispersity might become an important parameter.

Another common source of polydispersity is aggregation. Aggregation refers to the usually irreversible formation of large disordered and heterogeneous clusters, which have irregular and arbitrary shapes, sizes, and effective mass densities. ${ }^{[51-53]}$ The colloidal stability of NPs in suspension is controlled by interparticle interactions driven by intermolecular and surface forces. ${ }^{[54]}$ The balance of attractive and repulsive forces determines the dispersion state of the NPs and the fate of the colloidal suspension. A repulsive net force will ensure stability, while an unbalanced attractive net force will minimize the energy corresponding to the interaction potential: the colloidal suspension will collapse. ${ }^{[54,55]}$ As soon as NPs enter biological fluids, such as cell-culture medium, they find themselves in a complex matrix populated by bio- and small molecules, such as proteins, salts, vitamins, or lipids. ${ }^{[22]}$ These biomolecules can engage into specific interactions with the NPs, ${ }^{[34,47]}$ whose surfaces are subsequently modified by their adsorption, which affects colloidal behavior. ${ }^{[56-58]}$ Presently, our ability to predict NP behavior in a particular cell medium, with or without different concentrations of serum components, is limited, due to the complex character of the NPs and the media. ${ }^{[32,59]}$ Likewise, thorough and real-time analytical investigation of the state of dispersion during an experiment is challenging. ${ }^{[18,19]}$ It is conceivable that many colloidal suspensions will not remain stable and NPs will aggregate. ${ }^{[60-63]}$ Owing to the size, the transport of aggregates is generally governed by sedimentation.

To fill this knowledge gap existing for particokinetics and in vitro dosimetry, we isolate and demonstrate the characteristic features that emerge from polydispersity and present a new approach that reaches beyond uniform and instantaneously adhering particles (http://dyce.bionanomaterials.ch). The results presented in this report are strictly based on modeling and computation, which provides an ideal environment for systematic studies using well-controlled parameters and a userdefined complexity that can be gradually adapted. ${ }^{64,65]}$ Pure modeling and simulation is a deliberate choice of ours as direct experimental studies currently are not able to isolate and eliminate the influence of nanoparticle administration (i.e., pipetting) and nanoparticle detection. Most methods developed for detecting and quantifying nanoparticles are based on chemical elementary analysis [e.g., ICP techniques, ${ }^{[66,67]}$ optical spectroscopy (e.g., absorption, ${ }^{[68]}$ fluorescence, ${ }^{[69]}$ and vibrational ${ }^{[70,71]}$ spectroscopy), magnetic properties of the nanoparticle itself (e.g., superconducting quantum interference technique), ${ }^{[72]}$ a molecular probe associated to the NPs, or neutron activation (e.g., radiolabeling techniques)]. ${ }^{73-75]}$ Beside the current challenge of establishing a simple, highly reproducible, controllable, and last but not least easily accessible dose-delivery process, all these methods are not based on direct, primary measurable quantities (i.e., number of particles, overall mass, overall surface area) but on secondary quantities, such as the fluorescence from molecules associated to the nanoparticles, vibrational, emission or absorption spectra of NPs themselves, molecular probes attached, or the ratio of radioactive isotopes to stable isotopes in NPs. Consequently, one should describe the quantitative relationship between secondary measurable quantities and the primary quantities. However, even if it was done prior to experiment, secondary measurable quantities are prone to be influenced by external parameters: e.g., fluorescence intensity can vary due to both aggregation and photobleaching. The issues of particle administration and detection may bring in considerable uncertainty. Indeed, although several articles report a positive correlation between estimated and measured dose, ${ }^{[20-25]}$ their absolute value may differ up to several orders of magnitude, ${ }^{[23]}$ which strongly indicates that currently available and commonly used nanoparticle administration and detection methods rather allow describing only relative doses instead of absolute doses. ${ }^{[76]}$

Therefore, we restrict our study to heterogeneous yet welldefined scenarios, i.e., gold ( $\mathrm{Au}$ ) and polystyrene (PS) spherical NPs. We opted for these materials because of the high (Au: $19.2 \mathrm{~g} \mathrm{~cm}^{-3}$ ) and low (PS: $1.05 \mathrm{~g} \mathrm{~cm}^{-3}$ ) mass densities, respectively. Nonetheless, our method is modular and flexible and the selection we address here is not limited by the capabilities of the computational platform we developed, which can be used for a de facto broad range of particulate systems.

\section{Theory}

In a dilute quiescent colloidal suspension, there is no collective fluid motion. When viscous forces dominate over inertial forces and interparticle interactions are negligible, the kinetics of a particle is a function of two parameters: hydrodynamic radius $\left(r_{\mathrm{h}}\right)$ and mass density $(\rho)$. The particle motion is quantified by the diffusion coefficient (the Stokes-Einstein equation)

$D\left(r_{\mathrm{h}}\right)=\frac{k_{\mathrm{B}} T}{6 \pi \eta} \frac{1}{r_{\mathrm{h}}}$ 
and the settling velocity (the Stokes' law)

$$
V\left(r_{\mathrm{h}}\right)=\frac{2 \mathrm{~g}}{9 \eta} \times\left(\rho-\rho_{\mathrm{f}}\right) \times r_{\mathrm{h}}^{2}
$$

$\eta$ is the dynamic viscosity of the fluid, $T$ the temperature of the fluid, $k_{\mathrm{B}}$ the Boltzmann constant, $g$ the gravitational acceleration constant, and $\rho_{\mathrm{f}}$ the mass density of the fluid, respectively. ${ }^{[27,29,77]}$ In case of sedimentation, the displacement of the particle in the direction defined by gravity is proportional to the duration of motion: $x_{V}=V t$. Unlike sedimentation, diffusion is a stochastic process and the particle may move in any direction with equal probability. Accordingly, diffusion can be described only in terms of statistical moments, such as average displacement $\left\langle x_{D}\right\rangle=0$ and mean-squared displacement: $\left\langle x_{D}^{2}\right\rangle=2 D t$. When particle movement is unrestricted, the dispersion profile, i.e., the probability density for finding the particle at given $t$ time at a distance $x$ from its initial position $x_{0}$, follows a Gaussian distribution

$$
p(x, t)=\frac{1}{\sqrt{4 \pi D\left(r_{\mathrm{h}}\right) t}} e^{-\frac{\left(x-x_{0}-V\left(\mathrm{~m}_{\mathrm{n}}\right)\right)^{2}}{4 D\left(\mathrm{~m}_{\mathrm{h}}\right) t}}
$$

The center of $p(x, t)$ is equal to $x_{0}-V t$, while the width and amplitude of the Gaussian distribution are both functions of $D t$, as illustrated in Figure 1.

Dispersion in the hydrodynamic radii is described by the probability density function $p\left(r_{\mathrm{h}}\right)$, quantifying the relative likelihood to take on a given value. If $p\left(r_{\mathrm{h}}\right)$ is known, the probability density functions describing the distribution of the diffusion coefficients $p_{D}(D)$ and the settling velocities $p_{V}(V)$ can be estimated by the rule of transforming random variables. Let $\Phi$ describe a functional relationship between two variables $y$ and $x: y=\Phi(x)$ and $x=\Phi^{-1}(y)$, and let the probability density function describing the distribution of $x$ be known: $p_{x}(x)$. The aim is finding $p_{\gamma}(\gamma)$ for a given relationship described by $\Phi$. The probability density function describing the distribution of $y$ can be calculated by using the following formula ${ }^{[78]}$

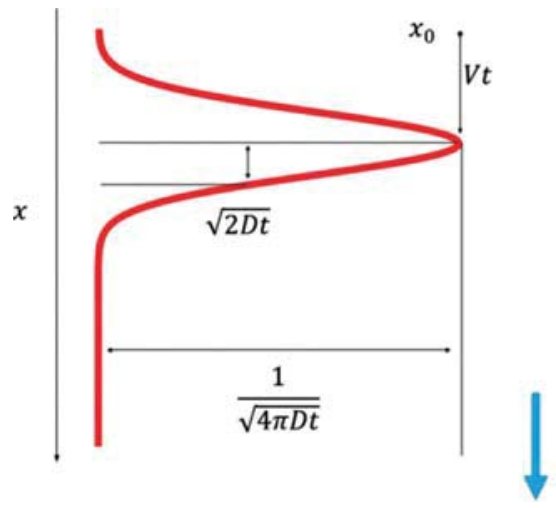

Figure 1. Characteristic features of the dispersion profile of a particle with $D$ diffusion coefficient and $V$ settling velocity. While the center of the Gaussian profile "travels" with a constant velocity $V$, the profile widens and its magnitude decreases in time with a $D$-dependent rate. The blue arrow indicates the direction of the gravitational force.
$p_{y}(\gamma)=p_{x}\left(\Phi^{-1}(\gamma)\right) \times\left|\frac{\mathrm{d}}{\mathrm{d} \gamma} \Phi^{-1}(\gamma)\right|$

To describe the hydrodynamic properties of polydisperse particles, let $\Phi$ embody the Stokes-Einstein equation and Stokes' law, respectively. Depending on particle type, e.g., concerning aerosols, powders, or engineered NPs, diverse mathematical models have been applied to describe the particle size distribution. Being one of the most common models, ${ }^{[79]}$ let the distribution of the hydrodynamic radius follow a lognormal distribution

$p\left(r_{\mathrm{h}}\right)=\frac{1}{\sqrt{2 \pi} r_{\mathrm{h}} \varepsilon} \mathrm{e}^{-\frac{\left(\ln r_{\mathrm{h}}-\mu\right)^{2}}{2 \varepsilon^{2}}}$

This distribution is described by two parameters: $\mu$ and $\varepsilon$, and it can be easily shown that the average hydrodynamic radius is given by $\left\langle r_{\mathrm{h}}\right\rangle=\int_{0}^{\infty} \mathrm{d} r_{\mathrm{h}} p\left(r_{\mathrm{h}}\right)=\mathrm{e}^{\mu+\frac{\varepsilon^{2}}{2}}$ and the polydispersity index $(\sigma=$ standard deviation divided by the mean) is $\mathrm{e}^{-\mu-\frac{\varepsilon^{2}}{2}} \sqrt{\mathrm{e}^{2 \mu+\varepsilon^{2}}\left(\mathrm{e}^{\varepsilon^{2}}-1\right)}$. The most probable value equal to the peak position of the distribution is $\mathrm{e}^{\mu-\varepsilon^{2}}$. Applying Equation (4), we obtain the probability density function of the diffusion coefficient

$p_{D}(D)=\frac{1}{\sqrt{2 \pi} D \varepsilon} \mathrm{e}^{-\frac{\left(\ln \frac{k_{1}}{D}-\mu\right)^{2}}{2 \varepsilon^{2}}}: k_{1}=\frac{k_{\mathrm{B}} T}{6 \pi \eta}$

as well as the probability density function of the settling velocity

$p_{V}(V)=\frac{1}{2 \sqrt{2 \pi} V \varepsilon} \mathrm{e}^{-\frac{\left(\ln \sqrt{\frac{V}{k_{2}}}-\mu\right)^{2}}{2 \varepsilon^{2}}}: \quad k_{2}=\frac{2 g}{9 \eta} \times\left(\rho-\rho_{\mathrm{f}}\right)$

We emphasize that (i) our approach is applicable for other probability density functions than lognormal, and (ii) the probability density function of the hydrodynamic radii can be also directly estimated by, e.g., dynamic light scattering. ${ }^{[0,81]}$ While the dispersion profile of uniform particles is purely Gaussian [Equation (3), Figure 1], in case of size polydispersity, the dispersion profile will be composed by the weighted contributions from each particle described in the distribution of the hydrodynamic radius $p\left(r_{\mathrm{h}}\right)$. Therefore, the dispersion profile of polydisperse particles is obtained by

$$
p(x, t)=\int_{0}^{\infty} \mathrm{d} r_{\mathrm{h}} p\left(r_{\mathrm{h}}\right) \times \frac{1}{\sqrt{4 \pi D\left(r_{\mathrm{h}}\right) t}} \mathrm{e}^{-\frac{\left(x-x_{0}-V\left(\eta_{\mathrm{h}}\right) t\right)^{2}}{4 D\left(\eta_{\mathrm{h}}\right) t}}
$$

\subsection{Random Walk Approach}

Random walk with a constant drift is a discrete representation of the particle's Brownian motion governed by diffusion and sedimentation. ${ }^{\left[{ }^{[2]}\right.}$ Random walk, mimicking particle motion, is a key technique in our approach.

In the discrete representation, the movement is not continuous in time (Figure 2). Instead, the particle performs a finite 


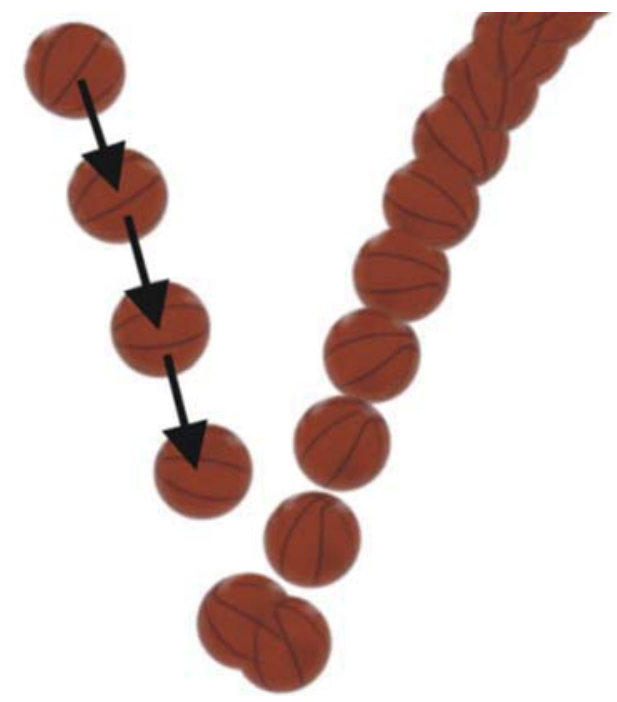

Figure 2. Random walk approach as illustrated by a stroboscopic image of a free-falling and bouncing basketball. The stroboscopic effect illustrates the concept of the random walk based on discrete steps: the sequence of the time-resolved observations is linked together by discrete steps indicated by the arrows, and although the representation is not continuous in time, the motion is faithfully captured.

number of $n=\omega t \gg 1$ steps in given time, where $\omega$ is referred to as the jumping frequency describing the number of steps the particle makes in unit time. The length of each step is chosen to be either $l_{+}=v+\delta$ or $l_{-}=v-\delta$, so that the chances for $l_{+}$and $l_{-}$ are equal and consecutive steps are uncorrelated. The term $\pm \delta$ represents diffusion, and $v$ represents a constant drift driven by sedimentation. Their value is calculated from the jumping frequency, diffusion coefficient, and the settling velocity

$\delta=\sqrt{2 D / \omega}$

$v=V / \omega$
In case of polydispersity, both $\delta$ and $v$ are polydisperse, and the steps of the random walk, $l_{+/-}$, are sampled over their distributions.

\subsection{The Initial and Boundary Conditions of the Random Walk}

The most common initial and boundary conditions are illustrated in Figure 3.

At the beginning, a given particle $\left(\mathrm{NP}_{\mathrm{i}}\right)$ is at a given position $h_{\mathrm{i}}$. At given time $t_{\mathrm{j}}$, the particle performs $\mathrm{n}$ steps. The walk obeys simple rules: the particle cannot cross the surface of the fluid (reflective boundary) and when the particle reaches the bottom, $\mathrm{h}_{\mathrm{i}}=\mathrm{H}$, it may adheres to cell (adsorbing boundary). We can also introduce a stochastic boundary, where upon reaching the cell, the particle may "dock" with probability $0<p<1$. Our primary interest is the particle's position after $\mathrm{n}$ steps: $h_{\mathrm{i}}(\mathrm{n})$ . Following the same rules, this walk is repeated $m \gg 1$ times, until statistically relevant estimation is obtained. Finally, we count that out of $\mathrm{m}$ walks how many have resulted in adhering to the bottom. This count estimates the fraction of particles that have been delivered to the cell from a given height $h_{\mathrm{i}}$ in given $t_{\mathrm{j}}$ time. If the particles are dispersed evenly in the beginning of the experiment (initial condition B), the walk described above must be performed and subsequently averaged over different heights.

\section{Results and Discussion}

\subsection{Influence of Polydispersity}

Figure 4 shows lognormal size distributions and corresponding dispersion profiles of small (10 nm) and large (100 nm) AuNPs. Clearly, the impact of polydispersity on the dispersion profile is different. For the small AuNPs, where diffusion is the dominating transport process, polydispersity has a moderate influence: the amplitude decreases and the tails of the profile become more elongated, resulting in a slight asymmetry. For large AuNPs, however, where the average size promotes settling
A

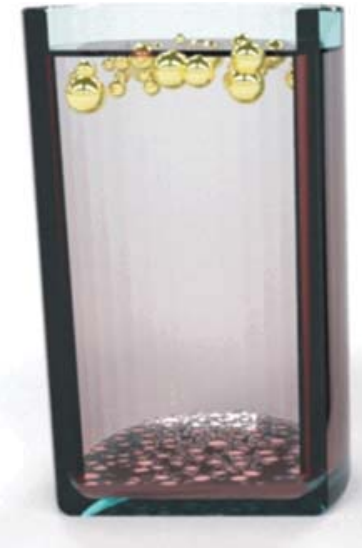

B

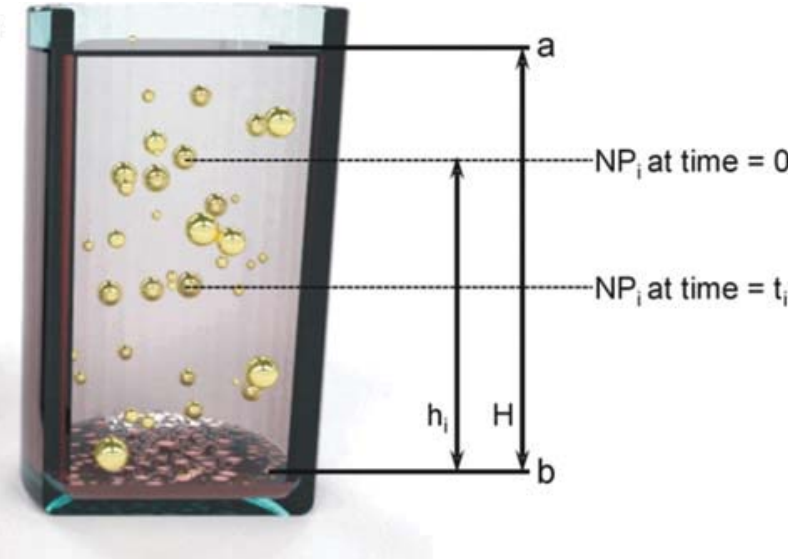

Figure 3. Initial and boundary conditions. Initial condition: one begins with particles being A) at the surface of the fluid of height $\mathrm{H}$, or B) homogenously dispersed in the fluid. Boundary conditions: during the experiment, the particle (a) cannot cross the surface of the fluid, and (b) when the particle reaches the cell, it may adhere to the cell. 
A: size distribution, AuNPs, $10 \mathrm{~nm}$

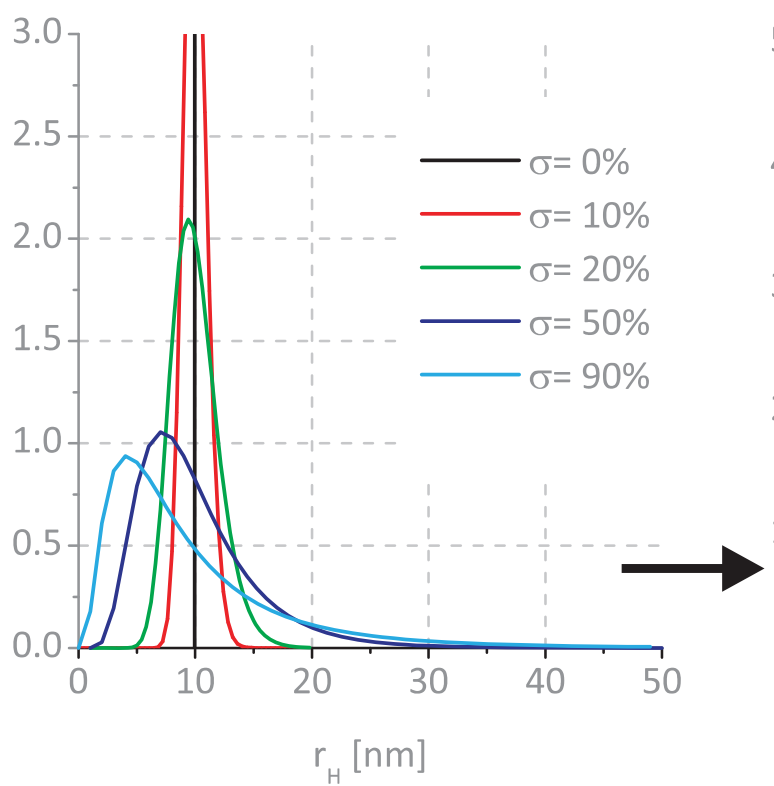

C: size distribution, AuNPs, $100 \mathrm{~nm}$

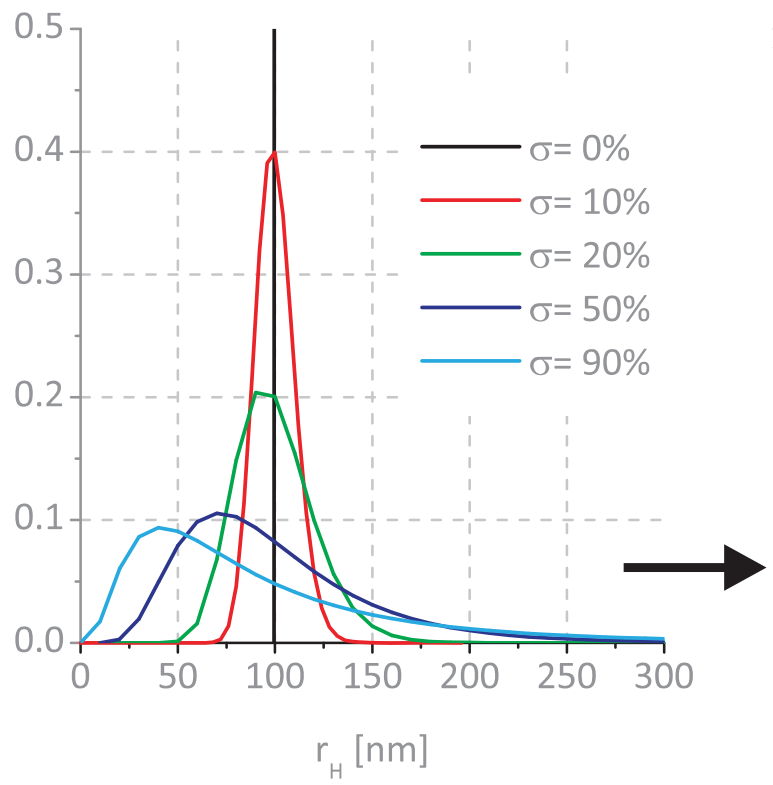

B: dispersion profile, AuNPs, $10 \mathrm{~nm}$

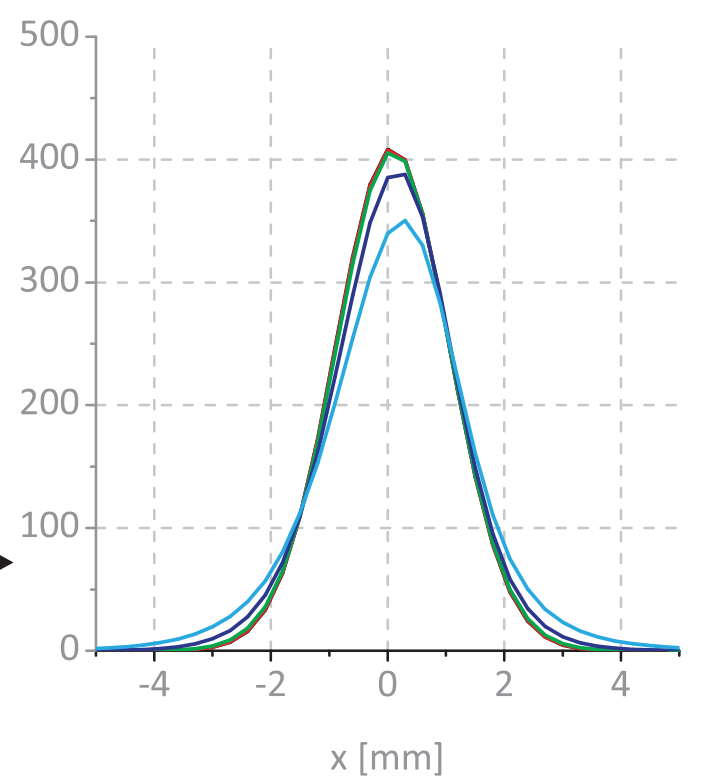

D: dispersion profile, AuNPs, $100 \mathrm{~nm}$

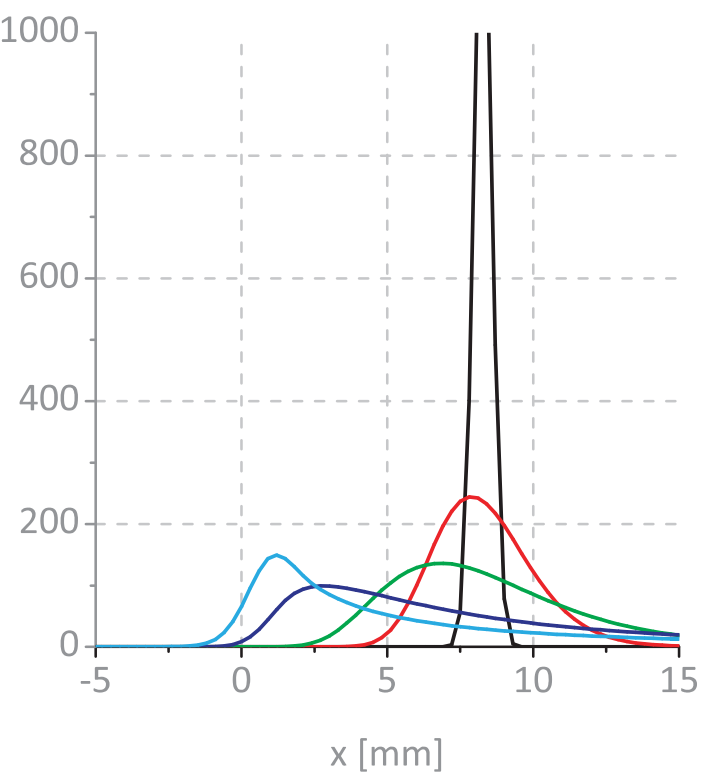

Figure 4. Particle size distributions and corresponding dispersion profiles of small and large AuNPs. Particle size distributions (lognormal, panels A and C) and corresponding dispersion profiles (panels B and D) of small and large AuNPs, respectively $\left(19.2 \mathrm{~g} \mathrm{~cm}^{-3}\right.$, time $=24 \mathrm{~h}$ at $\left.t=37{ }^{\circ} \mathrm{C}\right)$, with different degrees of polydispersity. The average hydrodynamic radius was kept constant in both cases. Polydispersity of the particle size distributions $(\sigma)$ is defined as the standard deviation over the mean.

over diffusion, polydispersity has important consequences: it results in a considerable deviation from the Gaussian profile of uniform AuNPs $(\sigma=0 \%)$, and further increase in polydispersity produces increasingly elongated and asymmetric profiles. These observations clearly demonstrate how size of polydispersity influences particle transport and that polydispersity cannot be neglected when transport is dominated by settling.
Since the dispersion profile is characteristic for the transport in general, it is expected to play a crucial role in the delivery of NPs to the surface of adherent cells in cell culture. Corresponding to typical in vitro conditions (Figure 5), the influence of polydispersity on the delivery-time course of heavy (i.e., Au) and light (i.e., PS) NPs following a lognormal size distribution is shown in Figure 6. 


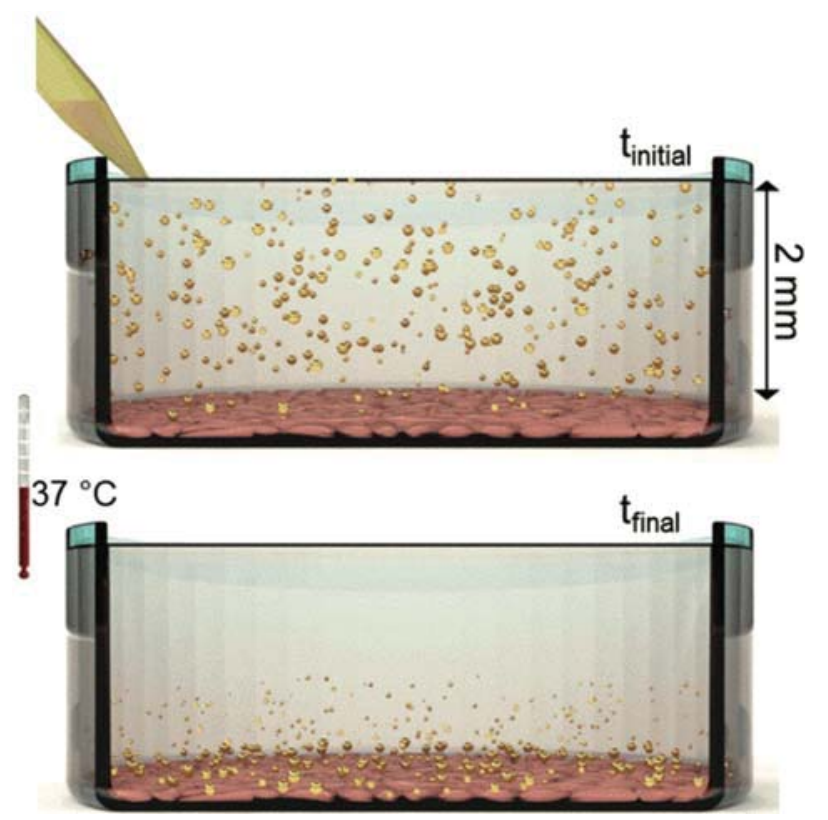

Figure 5. Illustration of in vitro cell-culture experiments and dosimetry. At the beginning $\left(t_{\text {initial }}\right)$, the particles are homogenously dispersed in the fluid, and at given time ( $\left.t_{\text {final }}\right)$ some of the particles, dictated by their dispersion profile, may become deposited. The diameter of the well and the overall volume of the administrated suspension will define the height of the media.

The selection of particle sizes took into account the availability and ease of published synthesis methods, ${ }^{[83-85]}$ and the synthesis of large (i.e., near-micron sized) AuNPs is rather challenging and relies on advanced non-standard synthetic procedures, ${ }^{[86]}$ and therefore, AuNPs up to $100 \mathrm{~nm}$ were considered. For small AuNPs, i.e., $10 \mathrm{~nm}$, where the average size promotes diffusion over settling, increasing $\sigma$ results in slightly higher delivery rates, though the increase is not significant below $\sigma=50 \%$ (Figure 6A). Accordingly even for high $\sigma$, the delivery-time course can be estimated using the average size. For large $(100 \mathrm{~nm})$ AuNPs (Figure 6B), where the average size promotes settling, the uniform AuNPs settle within $1 \mathrm{~h}$, the delivery-time curve rapidly saturates, and the NP suspension becomes depleted. Polydispersity, however, results in a smaller fraction of delivered NPs and clear differences between the time curves are present already above $\sigma=20 \%$. Some AuNPs reach the cell surface later than their uniform counterpart and at the highest $\sigma$, the delivered fraction is $40 \%$ below the expected average value (Figure 6B). A similar trend is observed for the much lighter PSNPs. For the smaller PSNPs (i.e., $100 \mathrm{~nm}$, Figure $6 \mathrm{C}$ ), where the average value favors diffusion over settling, increasing $\sigma$ again results in slightly higher delivery rates, though the increase is not notable below $\sigma=50 \%$. Therefore, estimating the delivery-time course by using only the average value is not critical, even when the polydispersity is considerable. When the average size promotes settling, the large (i.e., $1000 \mathrm{~nm}$ ) uniform PSNPs settle within $4 \mathrm{~h}$ and the delivery curve rapidly saturates (Figure 6D). Increasing $\sigma$ results in a smaller fraction of delivered particles and notable differences are present above $\sigma=20 \%$. Therefore, it is strongly indicated that when the average size promotes settling, the influence of size polydispersity should not be neglected, but rather must be incorporated when estimating the delivery-time course.

If the mass density of the NPs exceeds that of the fluid, the particles will sediment, and most particles will arrive at the bottom of the cell-culture well. When the fraction of particles deposited on the cell is equal to one, the suspension becomes depleted. Nonetheless, the time needed for depletion for a given average particle size is dependent on the polydispersity of the sample. A more detailed view of this dependence on polydispersity is shown in Figure 7, illustrating the fraction of delivered particles between 1 and $32 \mathrm{~h}$.

Even when considering NPs where transport is dominated by diffusion (i.e., $10 \mathrm{~nm}$ AuNPs and $100 \mathrm{~nm}$ PSNPs), a weak nonetheless increasing trend as a function of $\sigma$ is displayed. When the mean size promotes sedimentation, i.e., $100 \mathrm{~nm}$ AuNPs and $1000 \mathrm{~nm}$ PSNPs, increasing $\sigma$ results in a smaller delivered fraction. An important observation is that for a given particle, the influence of $\sigma$ is time-dependent. While the impact of $\sigma$ is most important at $1 \mathrm{~h}$ for the $100 \mathrm{~nm}$ AuNPs, a fourfold increase in incubation time $(t=4 \mathrm{~h})$ is necessary to show comparable data in the case of the $1000 \mathrm{~nm}$ PSNPs. This peculiarity is due to the nontrivial "competition" between diffusion and settling; sooner or later, one of the processes will dominate the transport, however, the time needed for reaching this state is quantified by the actual mean values of the diffusion coefficient and settling velocity.

To improve colloidal stability, allow for subsequent surface functionalization, or enhance, e.g., biocompatibility, NPs are frequently coated with synthetic or natural polymers. ${ }^{[87-89]}$ The polymer covering the NP forms a shell and defines the effective hydrodynamic radius. In general, polymeric coatings are flexible and can adopt different conformations, depending on the overall surface coverage and on the curvature of the NP surface. ${ }^{[90]}$ Consequently, surface coating adds further to the polydispersity of the particle, in particular if the molecular weight itself shows polydispersity. ${ }^{[49]}$ To gain insight into the characteristics of more realistic suspensions, we studied a system where both the particle core and polymer shell follow lognormal distributions (Figure 8A-B). Even if the core is practically monodisperse $(\sigma=10 \%)$, the effective mass density becomes polydisperse, and the distributions of the diffusion coefficient and settling velocity are jointly distributed (Figure 8D). We emphasize again that our concept is applicable with probability density functions other than lognormal.

There are numerous reports on NP behavior in cell-culture studies for application and toxicological evaluations, and it is not unusual that the colloidal stability of the suspension collapses in cell-culture media, and consequently, NPs aggregate. ${ }^{[36,91,92]}$ Aggregates frequently have a so-called mass-fractal structure and exhibit a striking structural feature called selfsimilarity: the nature of randomness and disorder may remain invariant over a wide range of length scales. ${ }^{[53,93]}$ In this case, the properties relevant for transport can be described by scaling concepts: the hydrodynamic radius and the effective mass density are functions of the fractal dimension $f_{\mathrm{d}}$ and aggregation number $N^{[94]}$ 
A AuNPs, $10 \mathrm{~nm}$

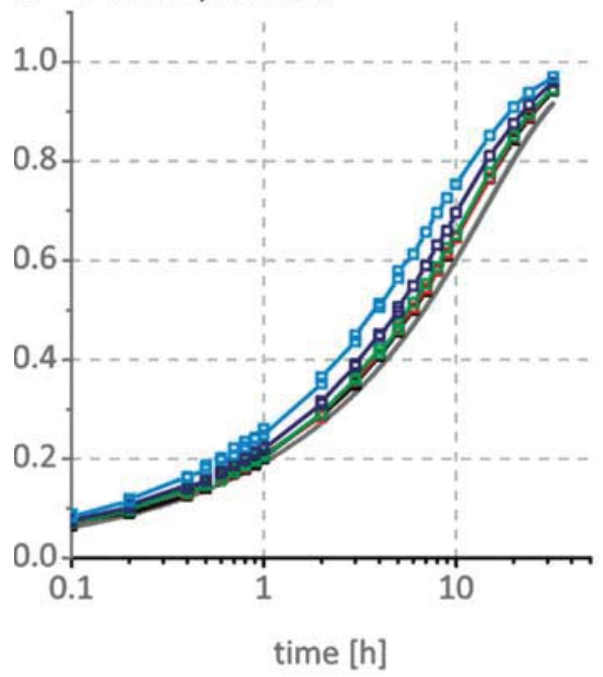

C PSNPs, $100 \mathrm{~nm}$

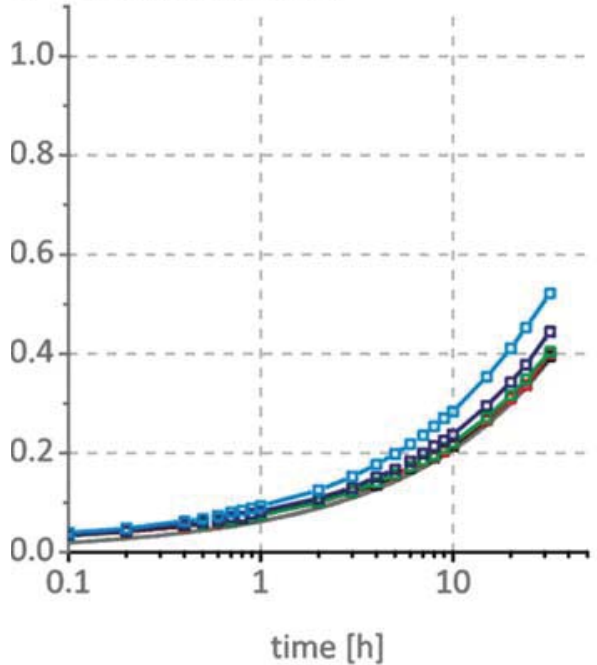

polydispersity $\sigma[\%]$
B AuNPs, $100 \mathrm{~nm}$

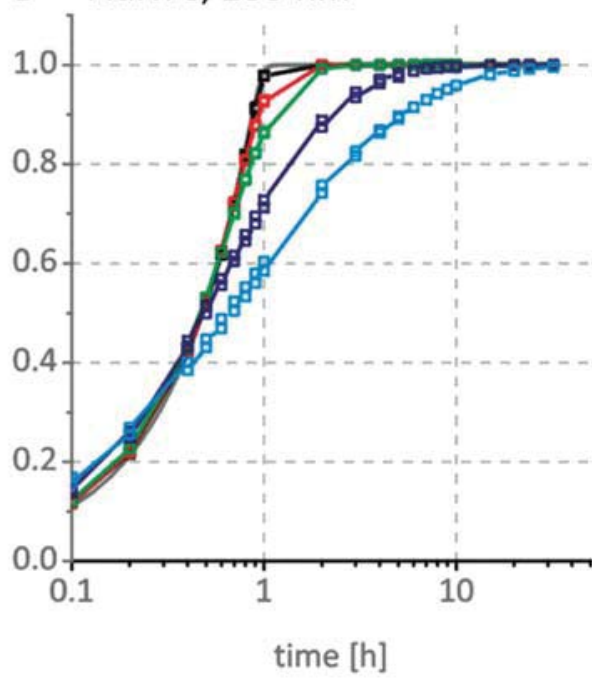

D PSNPs, $1000 \mathrm{~nm}$

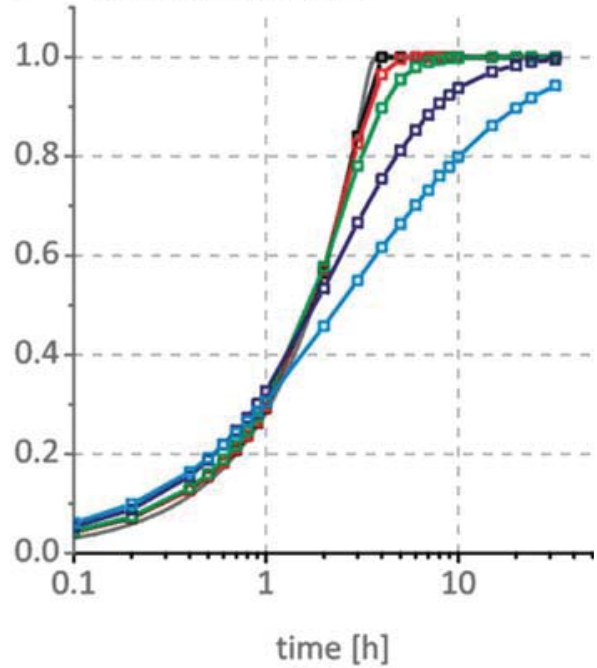

$$
0 \text { (ISDD) }-\mathbf{\square}-0-\square-10-\square-20-\square-50-\square-90
$$

Figure 6. Fraction of uniform and polydisperse particles delivered to the cell up to $32 \mathrm{~h}$ as a function of time. Top panels ( $A$ and $B$ ) show heavy particles (AuNPs, $19.2 \mathrm{~g} \mathrm{~cm}^{-3}$ ), and bottom panels (C and D) show light particles (PSNPs, $1.05 \mathrm{~g} \mathrm{~cm}^{-3}$ ). The average hydrodynamic radius is indicated for each panel. The height of the fluid medium was $2 \mathrm{~mm}$, and $t=37^{\circ} \mathrm{C}$. The simulation started with homogenously dispersed NPs, which could not cross the surface of the fluid and adhered instantaneously. In case of uniform particles $(\sigma=0 \%)$, the results given by the ISDD monodisperse mode ${ }^{[20]}$ and by our approach, respectively, are in agreement.

$$
r_{\mathrm{h}} \cong r \times N^{\frac{1}{f_{\mathrm{d}}}}
$$

where $r$ is the radius of the primary spherical particles, $N$ is the total number of the particles in the cluster, and $f_{\mathrm{d}}$ is the fractal dimension. The fractal dimension can be considered as a measure of the degree of packing of particles found in the aggregate. Since packing is never complete, the clusters also contain voids, and thus, the internal structure of an aggregate is porous. Due to the porous structure, the mass density of an aggregate is smaller than that of the primary particles. According to Equation (9), the mass density of the aggregate can be estimated as
$\rho_{\mathrm{R}}=\frac{\text { Total mass }}{\text { Hydr. volume }} \cong \frac{\rho \times N \frac{4}{3} \pi r^{3}}{\frac{4}{3} \pi r_{\mathrm{h}}^{3}}=\rho \times N^{1-\frac{3}{f_{\mathrm{d}}}}$

where $\rho$ is the mass density of the primary particles. Equation (10) indicates that at given $f_{\mathrm{d}}$, the larger the aggregate, the smaller the effective mass density, because the hydrodynamic volume of the aggregate grows at a higher rate than its total mass. In fluid, if the pores are completely filled with immobile fluid and Equation (10) is modified 


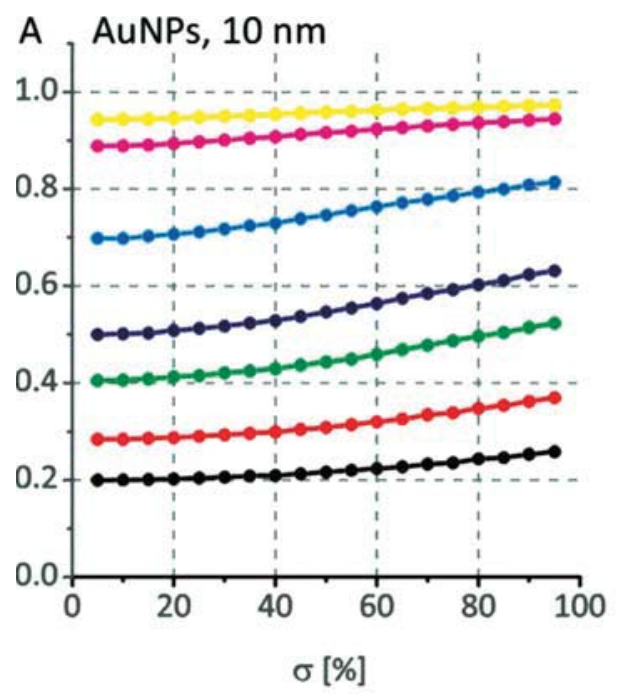

B AuNPs, $100 \mathrm{~nm}$

C PSNPs, $100 \mathrm{~nm}$

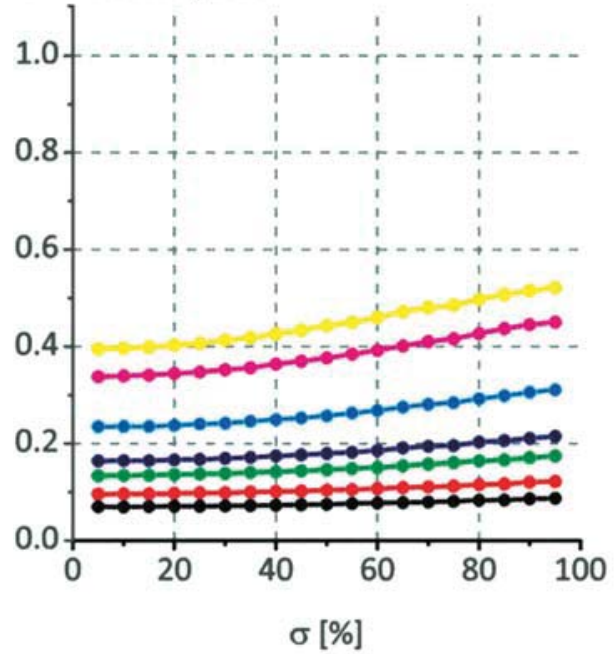

D PSNPs, $1000 \mathrm{~nm}$

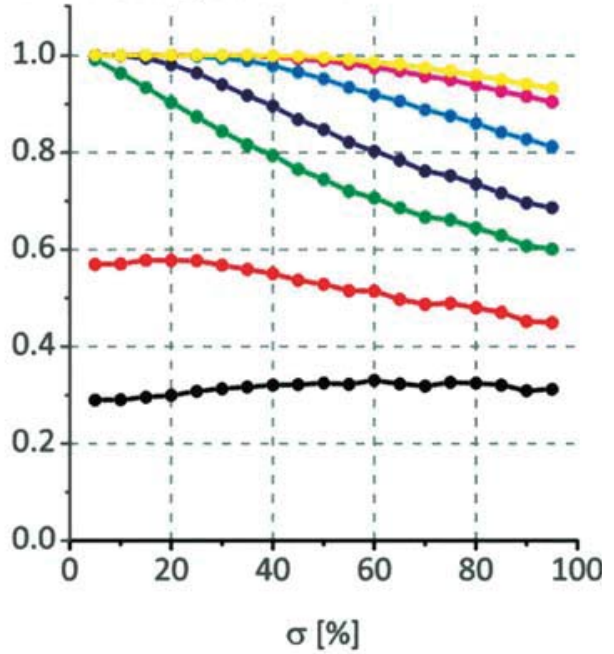

incubation time $[\mathrm{h}]$

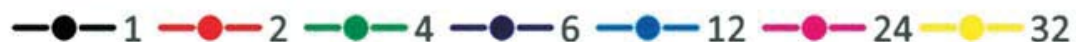

Figure 7. Fraction of particles delivered within different incubation times as a function of polydispersity. Top panels (A and B) show heavy particles (AuNPs), and bottom panels (C and D) show light particles (PSNPs). The average hydrodynamic radius is indicated for each panel. When diffusion is the dominating transport process, the impact is moderate, however, when sedimentation dominates the transport, the impact is pronounced upon increasing $\sigma$. The height of the fluid medium was $2 \mathrm{~mm}$, and $t=37^{\circ} \mathrm{C}$. The simulation begun with particles being homogenously dispersed in the fluid, and during the experiment the particles could not cross the surface of the fluid, and when the particle reached the cell it adhered instantaneously.

$\rho_{\mathrm{R}}^{\prime}=\rho_{\mathrm{R}}+\rho_{\mathrm{f}} \times\left(1-N^{1-\frac{3}{f_{\mathrm{d}}}}\right)$

From Equations (9-10), it follows that the effective mass density can be expressed as a function of the hydrodynamic radius and fractal dimension

$\rho_{\mathrm{R}}^{\prime}=\rho\left(\frac{r_{\mathrm{h}}}{r}\right)^{f_{\mathrm{d}}-3}+\rho_{\mathrm{f}}\left(1-\left(\frac{r_{\mathrm{h}}}{r}\right)^{f_{\mathrm{d}}-3}\right)$
The aggregation number $N$ as well as the fractal dimension $f_{\mathrm{d}}$ may vary from aggregate to aggregate, and hence, aggregates are not uniform but heterogeneous both in size and mass density, similarly to polymer-coated NPs. Figure 9 displays the heterogeneity of AuNP aggregates and the impact of aggregation on delivery. Aggregates are usually large, and thus, their diffusion coefficients are small and their transport is dominated by settling. Therefore, the impact of heterogeneity on delivery is critical (Figure 9D), and the average size cannot be used to estimate dose. 
A

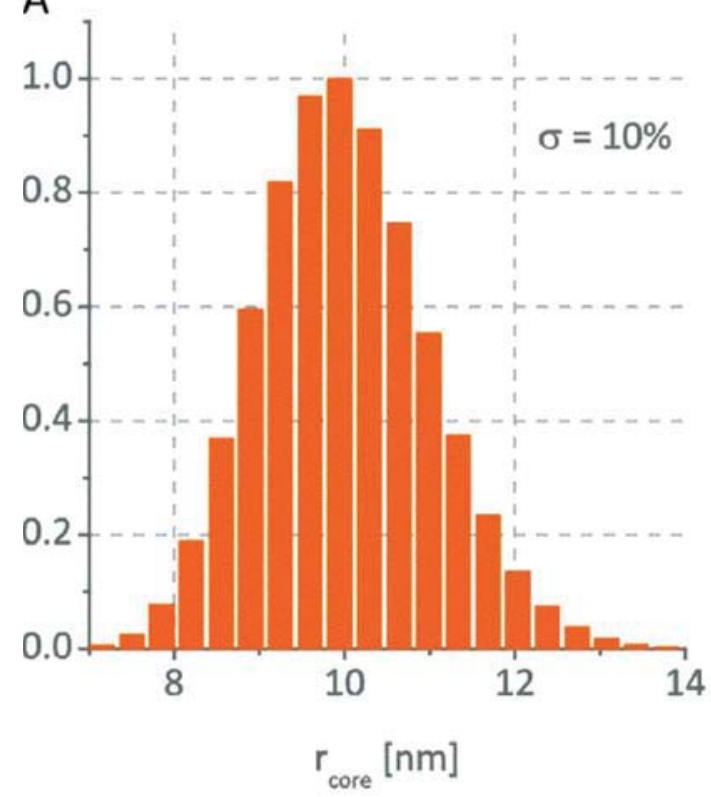

C

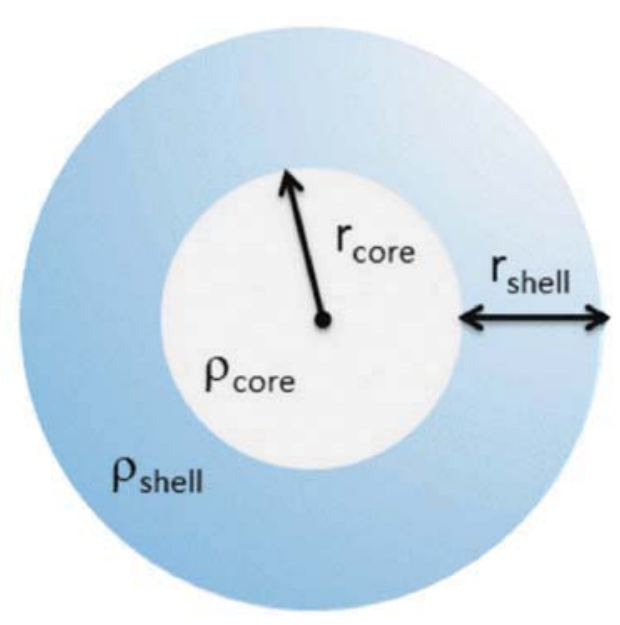

B

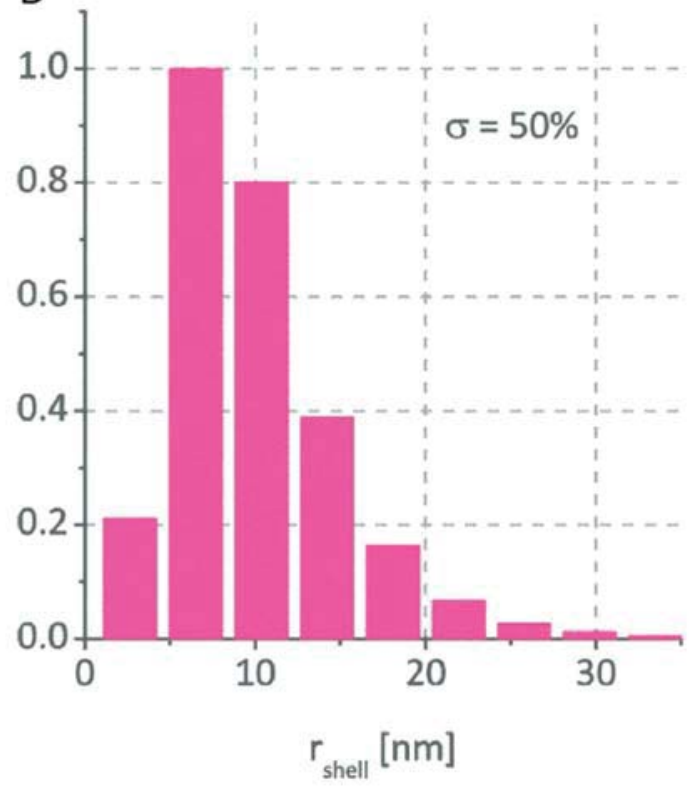

D

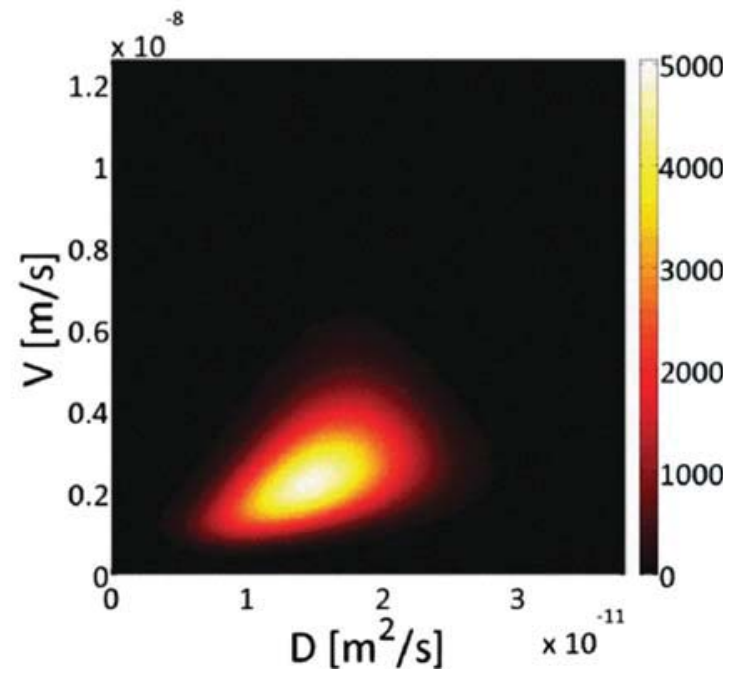

Figure 8. Heterogeneity of coated, core-shell like, particles. Panels A and B display the distributions of the core radius (gold, $19.2 \mathrm{~g} \mathrm{~cm}^{-3}$ ) and shell thickness (PEG, $1.1 \mathrm{~g} \mathrm{~cm}^{-3}$ ), respectively. Panel C illustrates the spherical core-shell model applied, and panel D displays the corresponding density map of the joint probability distribution of the diffusion coefficient and settling velocity.

\subsection{Influence of Non-Instantaneous NP Adherence}

Up to now, it has been postulated that upon reaching the cell, the NPs adhere instantaneously, resulting in an immediate association to the cell. However, it is known that at given surface chemistry the adhesion strength is actually size-dependent, ${ }^{[95]}$ and an immediate association cannot always take place. Once the NP reaches the cell surface, association is regulated by the chance that within a given $\Delta t$ time the particle adheres and binds to the cell. This chance may be expressed as a probability, ${ }^{[96]}$ i.e., when the particle reaches the cell, it may "dock" with probability $p$. Accordingly, the probability for not adhering is $1-p$. Depending on whether the particokinetics is dominated by diffusion or settling, this probability has consequences. If the particle does not adhere to the cell within $\Delta t$, the particle, instead of lingering nearby, may move away from the cell. A small particle can quickly diffuse away from the cell and this particle might need to "revisit" the cell more than once before actually "docking" to the cell surface. In contrast, a particle whose motion is dominated by settling will be kept by gravity near to the cell surface, standing by for adherence. Therefore, the residence time and particle mobility are expected to result in differences between diffusion dominated (10 nm AuNPs and $100 \mathrm{~nm}$ PSNPs) and large, sedimentation-dominated particles 
A

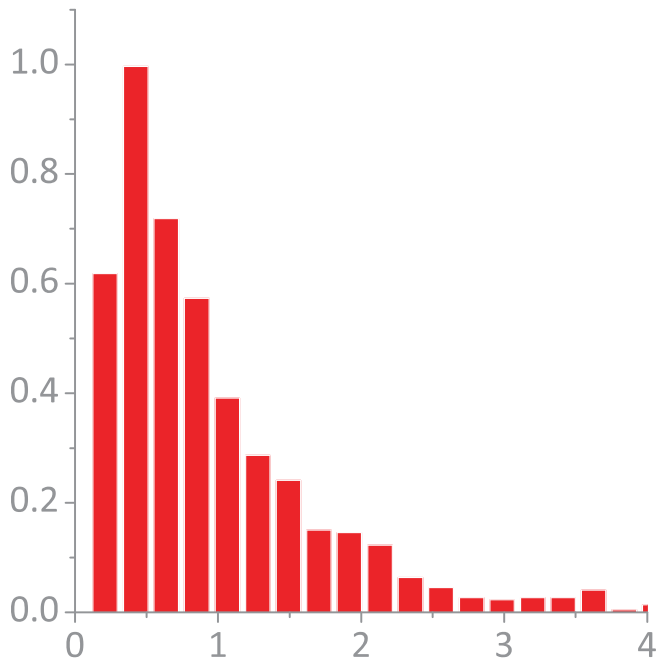

$\mathrm{N} / 10^{3}$

C

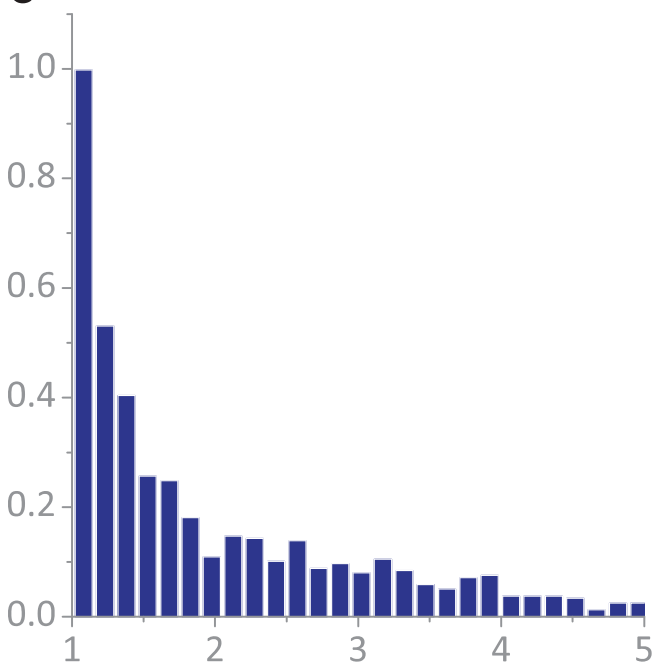

$\rho\left[\mathrm{g} / \mathrm{cm}^{3}\right]$
B

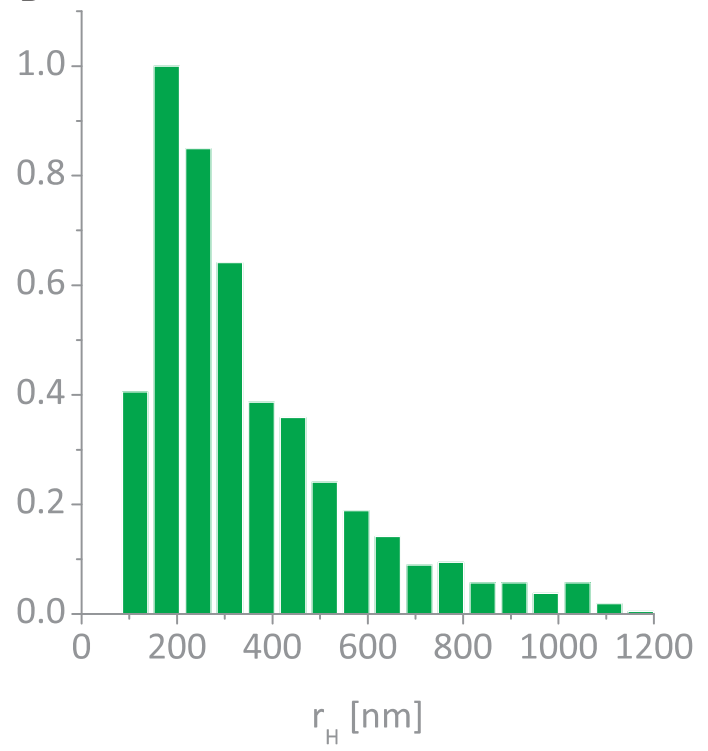

D

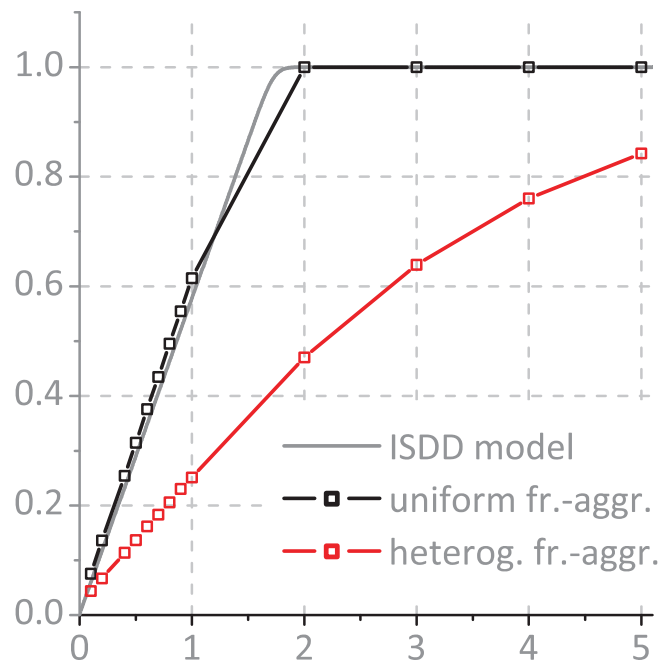

time $[h]$

Figure 9. Heterogeneity of nanoparticle fractal aggregates. Panel A displays the distribution of the number of uniform gold particles (10 $\mathrm{nm}$ radius) for an aggregate $\left(f_{\mathrm{d}}=2.1\right)$. Panels $\mathrm{B}$ and $\mathrm{C}$ display the resulting distributions of the hydrodynamic radius and effective mass density, respectively. Panel $\mathrm{D}$ shows that the impact of heterogeneity on delivery is critical. In case of uniform aggregates, the results given by the ISDD monodisperse model[20] and by our approach, respectively, are in agreement.

(100 nm AuNPs and $1000 \mathrm{~nm}$ PSNPs) (Figure 10). Polydispersity adds further to the complexity because the probability of adherence as well as the particle mobility is size-dependent.

\section{Conclusion}

We studied the influence of heterogeneity, including polydispersity both in size and mass density, on the dispersion profile of NPs in suspension experiments. Our study focused on (i) single, (ii) polymer-coated, and (iii) aggregated NPs and showed that when the average particle size promotes settling, polydispersity cannot be neglected in the estimation of the delivered dose as a function of time. This condition can be relaxed when the average particle size promotes diffusion. We also introduced a new concept where the adherence is probabilistic, and discussed the relevance: the influence of a non-instantaneous particle association to the cell is negligible for particles whose motion is dominated by settling, but it is relevant for small particles whose motion is governed by diffusion. To make the model of this study accessible, simulations can be run directly on a simple web interface 
A AuNPs, $10 \mathrm{~nm}$

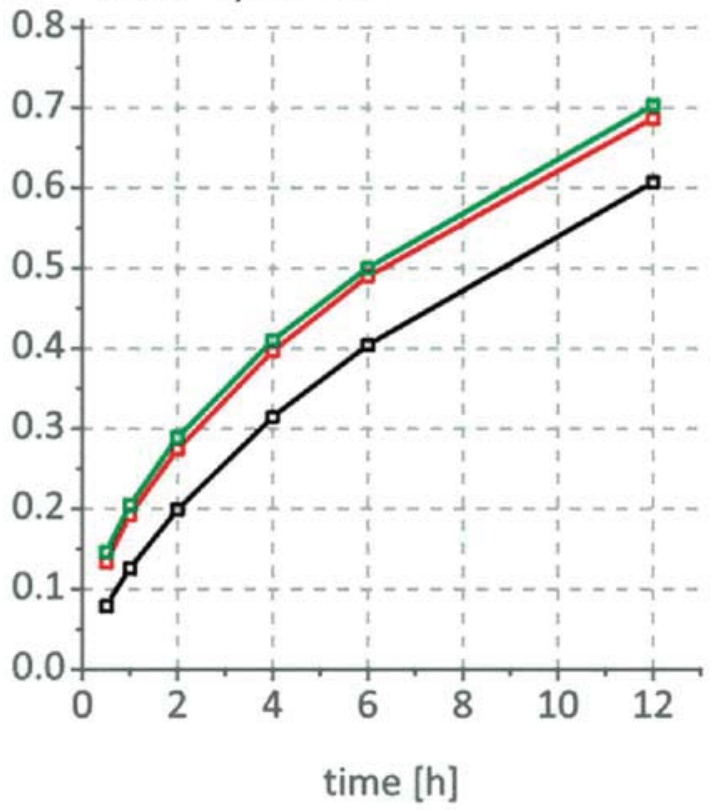

C PSNPs, $100 \mathrm{~nm}$

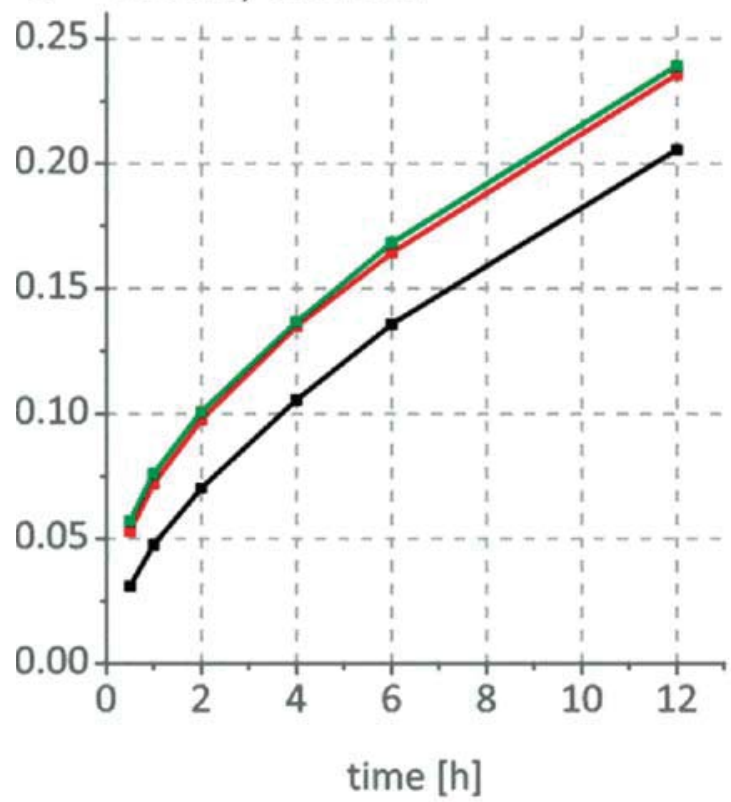

B AuNPs, $100 \mathrm{~nm}$

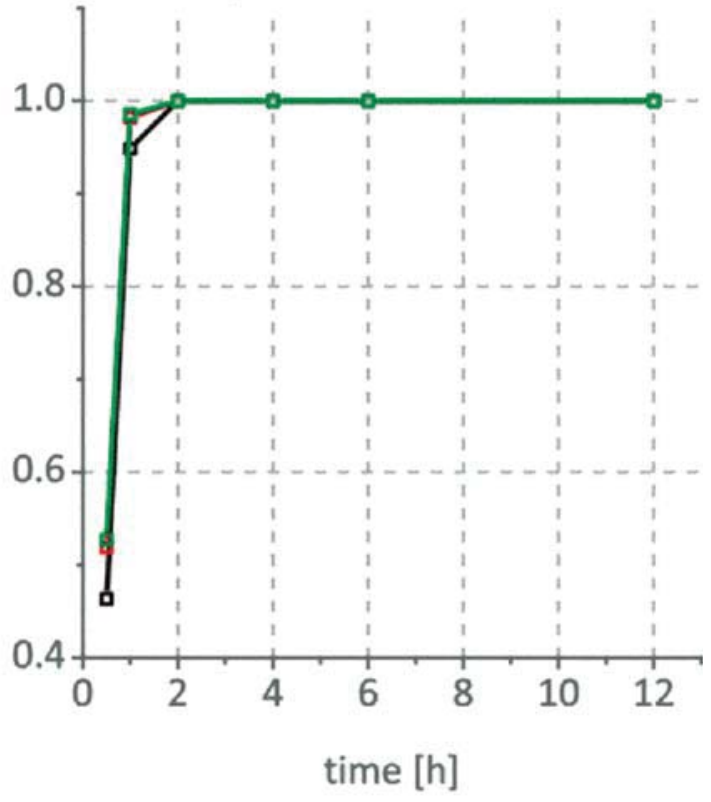

D PSNPs, $1000 \mathrm{~nm}$

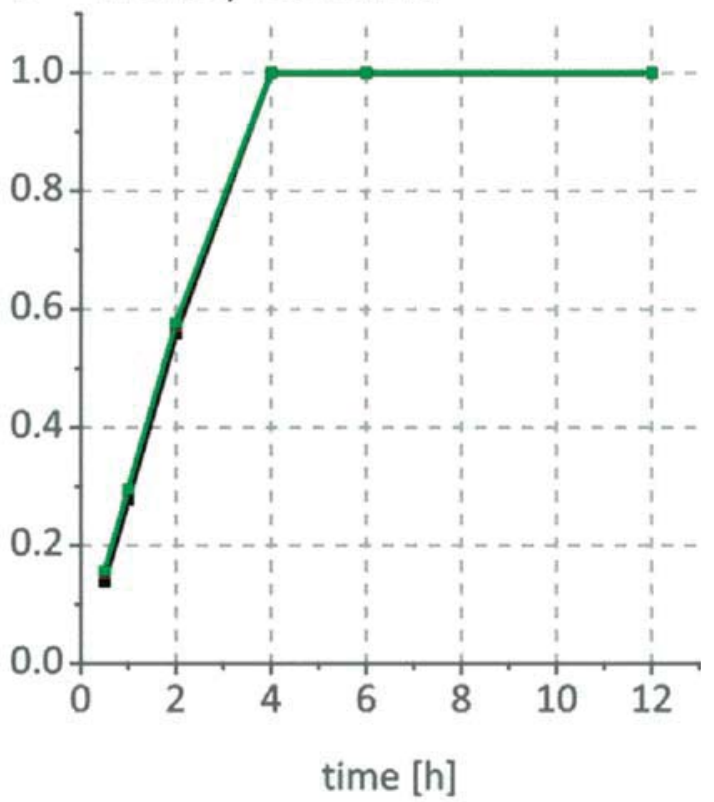

Probability of adherence

-"- $p=0.1-\backsim-p=0.5-m-p=1$

Figure 10. Fraction of uniform particles associated to the cell up to $12 \mathrm{~h}$ as a function of time, at different probabilities of adherence. Top panels (A and B) show heavy particles (AuNPs), and bottom panels (C and D) show light particles (PSNPs). The height of the fluid medium was $2 \mathrm{~mm}$, and $t=37^{\circ} \mathrm{C}$. The simulation begun with particles being homogenously dispersed in the fluid, and during the experiment the particles could not cross the surface of the fluid, and when the particle reached the cell it adhered with a probability $p$.

(http://dyce.bionanomaterials.ch). As many in vitro studies involve suspensions of heterogeneous NPs, such as commercially available NPs synthesized on an ultra-large scale, the distributions of the diffusion coefficient and the settling velocity as well as the corresponding particle transport profile must be considered. 


\section{Acknowledgements}

The authors are grateful for the financial support of the Adolphe Merkle Foundation and the Swiss National Science Foundation (APF: PP00P2$123373 / 1)$. Part of this research was supported by the Swiss National Science Foundation through the National Centre of Competence in Research Bio-Inspired Materials. L.R.-L. acknowledges the financial support from the L'Oreal Switzerland and UNESCO's fellowship program "For Women in Science 2013." The authors are grateful to Justin G. Teeguarden (Biological Sciences Division, Pacific Northwest National Laboratory, Richland, WA, USA) for providing the ISDD code (as of 14.12.2012) to compare the results for monodisperse NPs given by his and our computational platform. The authors are also grateful to Thomas L. Moore for reading carefully and commenting on the manuscript.

[1] S. M. Moghimi, A. C. Hunter, J. C. Murray, FASEB. J. 2005, 19, 311.

[2] K. Riehemann, S. W. Schneider, T. A. Luger, B. Godin, M. Ferrari, H. Fuchs, Angew. Chem Int. Ed. 2009, 48, 872.

[3] V. Wagner, A. Dullaart, A. K. Bock, A. Zweck, Nat. Biotechnol. 2006, 24, 1211 .

[4] L. Zhang, F. X. Gu, J. M. Chan, A. Z. Wang, R. S. Langer, O. C. Farokhzad, Clin. Pharmacol. Ther. 2008, 83, 761.

[5] N. Lewinski, V. Colvin, R. Drezek, Small 2008, 4, 26.

[6] M. A. Maurer-Jones, I. L. Gunsolus, C. J. Murphy, C. L. Haynes, Anal. Chem. 2013, 85, 3036.

[7] A. Maynard, D. Rejeski, Nature 2009, 460, 174.

[8] A. D. Maynard, R. J. Aitken, T. Butz, V. Colvin, K. Donaldson, G. Oberdorster, M. A. Philbert, J. Ryan, A. Seaton, V. Stone, S. S. Tinkle, L. Tran, N. J. Walker, D. B. Warheit, Nature 2006, 444, 267.

[9] G. Oberdorster, J. Intern. Med. 2010, 267, 89.

[10] S. Sharifi, S. Behzadi, S. Laurent, M. Laird Forrest, P. Stroeve, M. Mahmoudi, Chem. Soc. Rev. 2012, 41, 2323.

[11] S. E. Lohse, N. D. Burrows, L. Scarabelli, L. M. Liz-Marzan, C. J. Murphy, Chem. Mater. 2014, 26, 34.

[12] Y. Chen, H. R. Chen, J. L. Shi, Adv. Mater. 2013, 25, 3144.

[13] M. L. Personick, C. A. Mirkin, J. Am. Chem. Soc. 2013, 135, 18238.

[14] J. Wang, J. D. Byrne, M. E. Napier, J. M. DeSimone, Small 2011, 7, 1919.

[15] L. Zhang, W. F. Dong, H. B. Sun, Nanoscale 2013, 5, 7664.

[16] A. Albanese, W. C. W. Chan, ACS Nano. 2011, 5, 5478.

[17] E. C. Cho, Q. Zhang, Y. N. Xia, Nat. Nanotechnol. 2011, 6, 385.

[18] C. F. Jones, D. W. Grainger, Adv. Drug Delivery Rev. 2009, 61, 438.

[19] M. Horie, H. Kato, K. Fujita, S. Endoh, H. Iwahashi, Chem. Res. Toxicol. 2011, 25, 605 .

[20] P. M. Hinderliter, K. R. Minard, G. Orr, W. B. Chrisler, B. D. Thrall, J. G. Pounds, J. G. Teeguarden, Part. Fibre Toxicol. 2010, 7, 36.

[21] M. T. Zhu, S. Perrett, G. J. Nie, Small 2013, 9, 1619.

[22] J. Cohen, G. DeLoid, G. Pyrgiotakis, P. Demokritou, Nanotoxicology 2013, 7, 417

[23] R. A. Khanbeigi, A. Kumar, F. Sadouki, C. Lorenz, B. Forbes, L. A. Dailey, H. Collins, J. Controlled Release 2012, 162, 259.

[24] G. Sharma, V. Kodali, M. Gaffrey, W. Wang, K. R. Minard, N. J. Karin, J. G. Teeguarden, B. D. Thrall, Nanotoxicology 2014, 8, 663.

[25] J. G. Teeguarden, P. M. Hinderliter, G. Orr, B. D. Thrall, J. G. Pounds, Toxicol. Sci. 2007, 97, 614.

[26] R. Clift, J. R. Grace, M. E. Weber, Bubbles, Drops, and Particles, Academic Press, New York 1978.
[27] A. Einstein, Ann. Phys. Berlin 1905, 17, 549.

[28] E. M. Wray, Phys. Edu. 1977, 12, 300.

[29] M. von Smoluchowski, Ann. Phys. Berlin 1906, 326, 756.

[30] T. Sugimoto, Monodispersed Particles, Elsevier, Amsterdam 2001.

[31] A. Brunelli, G. Pojana, S. Callegaro, A. Marcomini, J. Nanopart. Res. 2013, 15, 1

[32] J. K. Jiang, G. Oberdorster, P. Biswas, J. Nanopart. Res. 2009, 11, 77.

[33] A. Kroll, M. H. Pillukat, D. Hahn, J. Schnekenburger, Eur. J. Pharm. Biopharm. 2009, 72, 370.

[34] A. E. Nel, L. Madler, D. Velegol, T. Xia, E. M. V. Hoek, P. Somasundaran, F. Klaessig, V. Castranova, M. Thompson, Nat. Mater. 2009, 8, 543.

[35] D. Walczyk, F. B. Bombelli, M. P. Monopoli, I. Lynch, K. A. Dawson, J. Am. Chem. Soc. 2010, 132, 5761.

[36] L. K. Limbach, Y. Li, R. N. Grass, T. J. Brunner, M. A. Hintermann, M. Muller, D. Gunther, W. J. Stark, Environ. Sci. Technol. 2005, 39, 9370.

[37] J. Schaefer, C. Schulze, E. E. J. Marxer, U. F. Schaefer, W. Wohlleben, U. Bakowsky, C.-M. Lehr, ACS Nano. 2012, 6, 4603.

[38] K. Wittmaack, ACS Nano. 2011, 5, 3766.

[39] R. C. Murdock, L. Braydich-Stolle, A. M. Schrand, J. J. Schlager, S. M. Hussain, Toxicol. Sci. 2008, 101, 239.

[40] M. P. Calatayud, C. Riggio, V. Raffa, B. Sanz, T. E. Torres, M. R. Ibarra, C. Hoskins, A. Cuschieri, L. Wang, J. Pinkernelle, G. Keilhoff, G. F. Goya, J. Mater. Chem. B 2013, 1, 3607.

[41] O. A. Sadik, Environ. Sci.: Proc. Impacts 2013, 15, 19.

[42] C. Buzea, I. I. Pacheco, K. Robbie, Biointerphases 2007, 2, Mr17.

[43] T. Kuhlbusch, C. Asbach, H. Fissan, D. Gohler, M. Stintz, Part. Fibre Toxicol. 2011, 8, 22.

[44] S. Jebors, S. Cecillon, C. Faye, C. Enjalbal, M. Amblard, A. Mehdi, G. Subra, J. Martinez, J. Mater. Chem. B 2013, 1, 6510.

[45] J. B. Haun, N. K. Devaraj, B. S. Marinelli, H. Lee, R. Weissleder, ACS Nano. 2011, 5, 3204.

[46] M. Lundqvist, J. Stigler, G. Elia, I. Lynch, T. Cedervall, K. A. Dawson, Proc. Natl. Acad. Sci. 2008, 105, 14265.

[47] M. Mahmoudi, I. Lynch, M. R. Ejtehadi, M. P. Monopoli, F. B. Bombelli, S. Laurent, Chem. Rev. 2011, 111, 5610.

[48] C. D. Walkey, J. B. Olsen, H. B. Guo, A. Emili, W. C. W. Chan, J. Am. Chem. Soc. 2012, 134, 2139.

[49] R. P. Carney, J. Y. Kim, H. Qian, R. Jin, H. Mehenni, F. Stellacci, O. M. Bakr, Nat. Commun. 2011, 2, 335.

[50] B. Pelaz, S. Jaber, D. J. de Aberasturi, V. Wulf, T. Aida, J. M. de la Fuente, J. Feldmann, H. E. Gaub, L. Josephson, C. R. Kagan, N. A. Kotov, L. M. Liz-Marzan, H. Mattoussi, P. Mulvaney, C. B. Murray, A. L. Rogach, P. S. Weiss, I. Willner,W. J. Parak, ACS Nano. 2012, 6, 8468.

[51] M. Y. Lin, H. M. Lindsay, D. A. Weitz, R. C. Ball, R. Klein, P. Meakin, Nature 1989, 339, 360.

[52] P. Meakin, Annu. Rev. Phys. Chem. 1988, 39, 237.

[53] P. Meakin, Adv. Colloid Interface Sci. 1988, 28, 249.

[54] R. Hughes, in Colloid Science: Principles, Methods and Applications, 2nd, (Ed: T. Cosgrove), Willey-Blackwell, Bristol UK 2010, Ch. 1

[55] M. Motornov, Y. Roiter, I. Tokarev, S. Minko, in Handbook of Surface and Colloid Chemistry, (Ed: K. S. Birdi), CRC Press, Parkway, NW, USA 2009, Ch. 5.

[56] C. Schulze, C. Schulze, A. Kroll, C. Schulze, A. Kroll, C.-M. Lehr, U. F. Schäfer, K. Becker, J. Schnekenburger, C. Schulze Isfort, R. Landsiedel, W. Wohlleben, Nanotoxicology 2008, 2, 51.

[57] J. Mejia, J.-P. Piret, F. Noël, B. Masereel, O. Toussaint, S. Lucas, J. Nanopart. Res. 2013, 15, 1.

[58] I. Lynch, K. A. Dawson, Nano Today 2008, 3, 40.

[59] M. Boström, D. R. M. Williams, B. W. Ninham, Phys. Rev. Lett. 2001, 87, 168103.

[60] M. Baalousha, Sci. Total Environ. 2009, 407, 2093. 
[61] M. Baalousha, A. Manciulea, S. Cumberland, K. Kendall, J. R. Lead, Environ. Toxicol. Chem. 2008, 27, 1875.

[62] X. Y. Liu, G. X. Chen, C. M. Su, J. Colloid Interface Sci. 2011, 363, 84.

[63] D. Zhou, Z. Ji, X. Jiang, D. R. Dunphy, J. Brinker, A. A. Keller, PLoS ONE 2013, 8, e81239.

[64] E. S. Allman, J. A. Rhodes, Mathematical Models in Biology - An Introduction, Cambridge University Press, New York, NY, USA 2004.

[65] P. Macheras, A. Iliadis, Modeling in Biopharmaceutics, Pharmacokinetics and Pharmacodynamics, Springer, New York, NY, USA 2006.

[66] L. Rodriguez-Lorenzo, K. Fytianos, F. Blank, C. von Garnier, B. Rothen-Rutishauser, A. Petri-Fink, Small 2014, 10, 1341.

[67] P. Krystek, J. Tentschert, Y. Nia, B. Trouiller, L. Noël, M. Goetz, A. Papin, A. Luch, T. Guérin, W. de Jong, Anal. Bioanal. Chem. 2014, 406, 3853.

[68] A. Galimard, M. Safi, N. Ould-Moussa, D. Montero, H. Conjeaud, J.-F. Berret, Small 2012, 8, 2036.

[69] C. Gottstein, G. Wu, B. J. Wong, J. A. Zasadzinski, ACS Nano. 2013, 7, 4933.

[70] N. M. S. Sirimuthu, C. D. Syme, J. M. Cooper, Anal. Chem. 2010, 82, 7369.

[71] V. Amendola, M. Meneghetti, S. Fiameni, S. Polizzi, G. Fracasso, A. Boscaini, M. Colombatti, Anal. Met. 2011, 3, 849.

[72] M. Lévy, C. Wilhelm, M. Devaud, P. Levitz, F. Gazeau, Contrast Media Mol. Imaging 2012, 7, 373.

[73] J. Roberts, J. Antonini, D. Porter, R. Chapman, J. Scabilloni, S.-H. Young, D. Schwegler-Berry, V. Castranova, R. Mercer, Part. Fibre Toxicol. 2013, 10, 5.

[74] D. H. Oughton, T. Hertel-Aas, E. Pellicer, E. Mendoza, E. J. Joner, Environ. Toxicol. Chem. 2008, 27, 1883.

[75] M. F. Hughes, T. C. Long, W. K. Boyes, R. Ramabhadran, Nanotoxicology 2013, 7, 1064.

[76] V. Hirsch, C. Kinnear, L. Rodriguez-Lorenzo, C. A. Monnier, B. Rothen-Rutishauser, S. Balog, A. Petri-Fink, Nanoscale 2014, 6, 7325.
[77] G. G. Hirsch, Collected Mathematical and Physical Papers, Cambridge University Press, New York, NY, USA 1880, p. 75.

[78] Z. Peyton, J. Peebles, Probability, Random Variables, and Random Signal Principles, McGraw-Hill, New York, NY, USA 1987.

[79] H. G. Merkus, Particle Size Measurements: Fundamentals, Practice, Quality, Springer, Paperback, NW, USA 2009.

[80] R. Pecora, J. Nanopart. Res. 2000, 2, 123.

[81] S. R. Aragon, R. Pecora, J. Chem. Phys. 1976, 64, 2395.

[82] P. N. Pusey, Science 2011, 332, 802.

[83] J. Rodriguez-Fernandez, J. Perez-Juste, F. J. G. de Abajo, L. M. Liz-Marzan, Langmuir 2006, 22, 7007.

[84] M. Pellach, J. Goldshtein, O. Ziv-Polat, S. Margel, J. Photoch. Photobio. A 2012, 228, 60.

[85] J. S. Song, M. A. Winnik, Macromolecules 2005, 38, 8300.

[86] L. S. Live, M. P. Murray-Methot, J. F. Masson, J. Phys. Chem. C 2009, 113, 40.

[87] J. H. Lee, M. A. Mahmoud, V. Sitterle, J. Sitterle, J. C. Meredith, J. Am. Chem. Soc. 2009, 131, 5048.

[88] J. Manson, D. Kumar, B. J. Meenan, D. Dixon, Gold Bull. 2011, 44, 99.

[89] K. Rahme, L. Chen, R. G. Hobbs, M. A. Morris, C. O'Driscoll, J. D. Holmes, RSC Adv. 2013, 3, 6085.

[90] H. D. Hill, J. E. Millstone, M. J. Banholzer, C. A. Mirkin, ACS Nano. 2009, 3, 418.

[91] S. Lazzari, D. Moscatelli, F. Codari, M. Salmona, M. Morbidelli, L. Diomede, J. Nanopart. Res. 2012, 14, 920.

[92] L. V. Stebounova, E. Guio, V. H. Grassian, J. Nanopart. Res. 2011, 13, 233.

[93] R. Botet, R. Jullien, Phase Transit. 1990, 24-26, 691.

[94] M. Lattuada, H. Wu, M. Morbidelli, J. Colloid Interface Sci. 2003, 268, 96.

[95] H. Y. Yuan, J. Li, G. Bao, S. L. Zhang, Phys. Rev. Lett. 2010, 105, 138101

[96] C. Cozensroberts, D. A. Lauffenburger, J. A. Quinn, Biophys. J. 1990, 58,841 . 